“C 2016 IEEE. Personal use of this material is permitted. Permission from IEEE must be obtained for all other uses, in any current or future media, including reprinting/republishing this material for advertising or promotional purposes, creating new collective works, for resale or redistribution to servers or lists, or reuse of any copyrighted component of this work in other works." 


\title{
Design and Testing of Simple, Electrically Small, Low-Profile, Huygens Source Antennas with Broadside Radiation Performance
}

\author{
Ming-Chun Tang, Senior Member, IEEE, Hao Wang, Student Member, IEEE, and Richard W. \\ Ziolkowski, Fellow, IEEE
}

\begin{abstract}
The efficacy of a simple, electrically small, low-profile, Huygens source antenna that radiates in its broadside direction is demonstrated numerically and experimentally. First, two types of electrically small, near field resonant parasitic (NFRP) antennas are introduced and their individual radiation performance characteristics are discussed. The electric one is based on a modified Egyptian axe dipole NFRP element; the magnetic one is based on a capacitively loaded loop (CLL) NFRP element. In both cases the driven element is a simple coax-fed dipole antenna, and there is no ground plane. By organically combining these two elements, Huygens source antennas are obtained. A forward propagating demonstrator version was fabricated and tested. The experimental results are in good agreement with their analytical and simulated values. This low profile, $\sim 0.05 \lambda_{0}$, and electrically small, $k a=0.645$, prototype yielded a peak realized gain of $2.03 \mathrm{dBi}$ in the broadside direction with a front-to-back ratio (FTBR) of $16.92 \mathrm{~dB}$. A backward radiating version is also obtained; its simulated current distribution behavior is compared to that of the forward version to illustrate the design principles.
\end{abstract}

Index Terms - Broadside directivity, electrically small antennas, Huygens source, low profile, metamaterial-inspired structures, near field resonant parasitic antennas.

\section{INTRODUCTION}

$\mathrm{B}$ ecause of their compact sizes preferred in many wireless communication platform designs, electrically small antennas (ESAs) have been extensively studied over the last

Manuscript received on Jan. 19, 2016, revised on Jul. 27, and accepted on Sep. 02, 2016.

This work was supported in part by the National Natural Science Foundation of China contract number 61471072, in part by graduate scientific research and innovation foundation of Chongqing, China contract numbers CYS16020, in part by the Fundamental Research Funds for the Central Universities contract number 106112015CDJZR165510, in part by China Postdoctoral Science Foundation contract number 2016M590860, and in part by Opening subject of State Key Laboratory of Millimeter Waves contract number K201732.

M. -C. Tang is with the College of Communication Engineering, Chongqing University, Chongqing, 400044, China and with the State Key Laboratory of Millimeter Waves, Nanjing, 210096, China (E-mail: tangmingchunuestc@126.com);

$\mathrm{H}$. Wang is with the College of Communication Engineering, Chongqing University, Chongqing, 400044, China;

R. W. Ziolkowski is with the Department of Electrical and Computer Engineering, University of Arizona, Tucson, AZ 85721 USA and with the University of Technology Sydney, Global Big Data Technologies Centre, Ultimo NSW 2007, Australia (E-mail: ziolkowski@ece.arizona.edu). few decades. In order to attain many of the desired radiation performance characteristics that are suitable for numerous wireless applications, there has been a great diversity of ESA designs reported with a variety of standard and more exotic configurations and operational mechanisms [1]-[9]. Nonetheless, because of their small electrical sizes, their directional radiation performance is constrained by basic physics [10], [11] to be electric or magnetic dipole-like. This means their peak realized gains and front-to-back ratios (FTBRs) are generally quite low. Considerable effort has been expended on improving the directional performance of ESAs. Higher directivity, efficient ESAs would be advantageous for many practical engineering applications, e.g., for long-distance and point-to-point wireless communications [12]-[13]; RFIDs [6], [14], [15]; and wireless power transfer (WPT) [16]-[19]. The common existing approaches can generally be classified into the following three categories. The first is the placement of an effective medium beneath the radiator that acts as an in-phase reflector, such as reactive impedance surface (RIS) substrates [20]; electromagnetic band gap (EBG) structures [21], [22]; and meanderline-shaped slot-structured conductor discs [23], [24]. The second is the arrangement of two, three or more radiators with similar structures, e.g., monopoles [25]-[29]; electrical dipoles [30], [31]; magnetic dipoles [32]; and ESAs [33], [34]; into an array to realize quasi-Yagi endfire configurations. The third is the construction of Huygens sources using pairs of electric and magnetic resonators/dipoles [35]-[38].

Comparing their physical operating mechanisms and physical structures, the Huygens source approach has distinct advantages over the other two [39]. For instance, increasing the number of radiating elements to form an array increases the overall length of the antenna system [25]-[34]. Furthermore, augmenting a radiator with periodic structures increases both its transverse size and height [20]-[24]. In contrast, electrically small, low profile, broadside radiating versions, which radiate preferentially into a hemisphere opposite the platform they are on, would be beneficial to many IoT (internet of things) wireless applications, e.g., on-body devices, RFIDs, WPT or harvesting systems, and environmental control and monitoring (sensor) systems.

Nevertheless, simple Huygens source ESA designs are difficult to realize in practice. Commonly, one would resort to combinations of completely separate electrical and magnetic dipoles, i.e., each radiator has its own input port and, hence, 
potentially a different input impedance matching requirement, and would attempt to achieve a certain degree of isolation between them [33], [35], [37], [38]. The end result is bulky and requires an additional design step - the alignment of the phase centers of the two radiators. Single feed designs have been successfully obtained [36], [40]-[43]. However, each has a certain drawback which restricts its widespread engineering application. On the one hand, the printed antenna designs radiate preferentially along the substrate, leading to a high profile along the mainbeam direction $\left(\sim 0.15 \lambda_{0}\right.$, where $\lambda_{0}=\mathrm{c} / f_{0}$ is the free space wavelength corresponding to the frequency of operation: $f_{0}$ ) [36]. On the other hand, the magnetoelectric dipole antennas [40]-[43], due to the inherent physical mechanisms of each of their electric and magnetic dipole combinations and the presence of the associated ground plane, inescapably make their electrical size not small (i.e., $k a=$ $2 \pi a / \lambda_{0}>1.0$, where $a$ is the radius of the smallest sphere that completely encloses the entire antenna system at $\lambda_{0}$ [9]). For example, the indispensable large ground plane needed to decrease the patch size and produce the equivalent magnetic dipole behavior in $[41,43]$ yields an antenna that has $k a \sim 5.91$; and the requisite $180^{\circ}$ phase delay between the two slots of the antenna reported in [42] makes the entire system $k a \sim 2.66$.

While it has been recently demonstrated numerically that extremely low-profile Huygens sources can be obtained [44], the reported designs are possible, but are complicated. Their configurations require multiple metamaterial unit cells which include lumped elements, making them difficult to fabricate and test. Moreover, because of the presence of the multiple near-field resonant parasitic (NFRP) elements, their Q-factors are increased significantly; and, hence, their $-10 \mathrm{~dB}$ fractional impedance bandwidths are quite small $(\sim 0.06 \%)$.

In this paper, based on the concepts reported in [44], a simple, low-profile, single-feed, broadside radiating, high-FTBR, Huygens source ESA is demonstrated numerically and experimentally. First, both the electric and magnetic NFRP dipole ESAs from which it is derived are introduced in Section II. These metamaterial-inspired ESA designs consist of a coax-fed dipole and, respectively, a modified Egyptian axe dipole (EAD) NFRP element and a capacitively loaded loop (CLL) NFRP element. The performance characteristics of both of these ESAs are investigated in detail. With $k a=2 \pi a / \lambda_{0}<$ 1.0 , each of these electrically small antennas radiates as an elemental dipole. They are organically combined in Section III to form the desired single-feed, low-profile Huygens source ESA. The resulting performance characteristics are obtained. A forward radiating demonstrator was fabricated and tested. The experimental results will be shown to be in good agreement with their simulated values. This prototype antenna was electrically small, $k a=0.645$, and low-profile with its height being $\sim \lambda_{0} / 20.45$. It produced a $2.03 \mathrm{dBi}$ peak realized gain and a $16.92 \mathrm{~dB}$ FTBR value. Its simulated directivity is $4.70 \mathrm{dBi}$, which was very close to the maximum analytical value: 4.77 $\mathrm{dBi}$ [36]. In contrast to the electrically large Huygens source antennas reported in [41] and [43] which have a $1.33 \times 1.33 \lambda_{0}{ }^{2}$ radiating cross-section, the one introduced here is only $0.0329 \lambda_{0}^{2}$ in transverse size, a 53.80 times reduction. Similarly, in contrast to the electrically large magnetoelectric dipole antenna reported in [42] whose radiating cross-section is
$0.63 \times 0.41 \lambda_{0}^{2}$, it is 7.86 times smaller.

In order to further investigate and illustrate the physical mechanisms of its Huygens source operation, a backward radiating version of the demonstrator was simulated. Comparisons of its current distributions with those of the forward radiating version help to further explain its operation. These design and operating principles are discussed in Section IV. Finally, conclusions are drawn in Section V.

We note that all of the metallic elements in the antenna designs reported in this paper were chosen to be copper with its known material parameters: $\varepsilon_{r}=1.0, \mu_{r}=0.999991$ and bulk conductivity $\sigma=5.8 \times 10^{7}$ Siemens $/ \mathrm{m}$. Similarly, the copper-cladded board material used was Rogers Duroid ${ }^{\mathrm{TM}} 5880$ with $0.5 \mathrm{oz}(17 \mu \mathrm{m})$ thick copper. All of the numerical simulations and their optimizations were performed using the frequency domain ANSYS/ANSOFT High Frequency Structure Simulator (HFSS), version 13 [45]. An operational frequency near $1.5 \mathrm{GHz}$ was selected for the design to ease the fabrication of the prototype and to match the available measurement equipment.

\section{ELECTRICALLY SMALL NFRP DIPOLE ANTENNAS}

A Huygens source ESA system can be achieved by properly combining electrically small electric and magnetic NFRP antennas [36], [44]. The metamaterial-inspired approach combines an electrically small resonator, i.e., the NFRP element, with a driven antenna. The NFRP element thus becomes the main radiator and the combination is tuned to achieve nearly complete matching to a $50 \Omega$ source. Thus, a modified EAD NFRP element is first combined with a coax-fed dipole antenna to achieve the electric piece. Then a CLL element is combined with the coax-fed dipole to achieve the magnetic piece separately. Their individual physical mechanisms and radiation characteristics are discussed in detail in preparation for their eventual combination.

\section{A. Electrically small electric dipole antenna}

Fig. 1 presents the evolution of the electrically small electric dipole antenna. First, the traditional center-fed half-wavelength dipole antenna, together with its surface current distribution and radiation pattern at its resonance frequency, is illustrated in Fig. 1(a). Since it is operating in its fundamental mode, the total length of dipole is approximately a half wavelength $\left(\lambda_{0} / 2\right)$ [46]. The current distribution shown in Fig. 1(a) is along the length of the dipole, i.e., along the $X$-axis on the metallic surfaces. The characteristic three-dimensional (3D) "electric dipole" directivity pattern (i.e., bi-directional in the $\mathrm{ZOX}(E)$-plane and omni-directional in the $\mathrm{ZOY}(H)$-plane [46]) is also shown.

Second, as is shown in Fig. 1(b), a typical EAD NFRP element is placed in the near field of the electrically small center-fed dipole antenna. It is designed in such a manner that the antenna system exhibits high radiation efficiency and good impedance matching to the $50 \Omega$ source without any external matching network, even though the complete antenna system is electrically small in size: $k a \sim 0.63$. The operating principles of this EAD NFRP antenna have been extensively explained in previous works [9], [22], [23], [31], and [47]. The surface current distributions on the metal surfaces are shown at the resonance frequency. As expected from any NFRP design [9], 
they are primarily on the NFRP element. Its 3D directivity pattern indicates that it too is radiating as an electric dipole oriented along the $X$-axis.

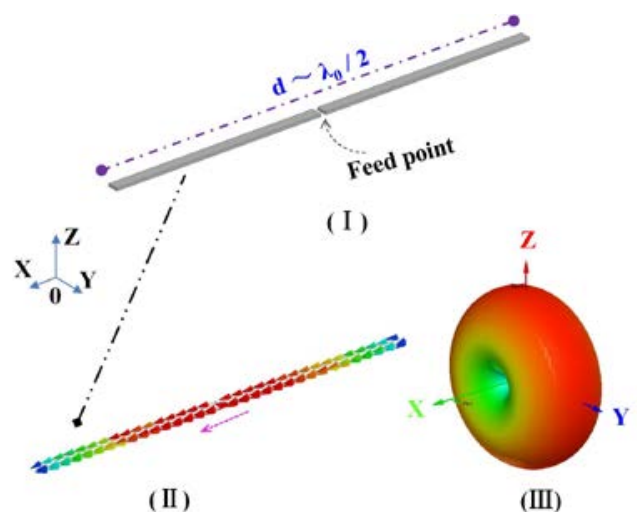

(a)



(b)

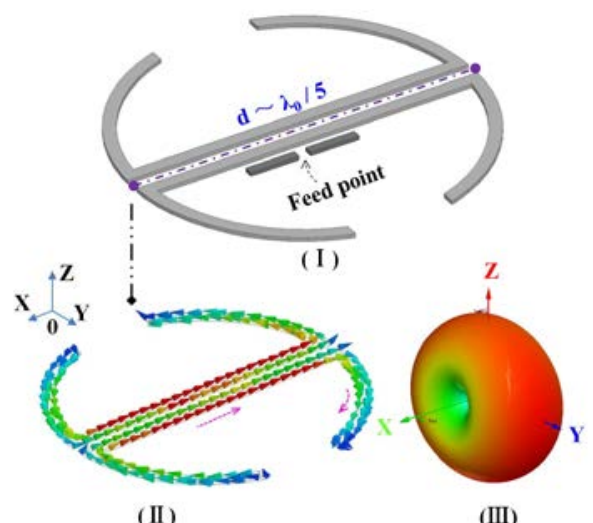

(c)

Fig. 1 The geometries, surface current distributions, and 3D directivity patterns for three electric dipole antennas: (a) traditional half-wavelength dipole, and electrically small (b) Egyptian axe and (c) modified Egyptian axe dipoles.

Third, as shown in Fig. 1(c), the EAD NFRP element can be split symmetrically along the $X$-axis into two parts. As will be recognized in the Huygens source antenna configuration, this design choice facilitates the requisite overlap of the phase centers of the electric and magnetic dipole radiators [35]-[37], [44] by providing space in which the magnetic NFRP element can be placed. It also provides more tuning degrees of freedom. Simulations indicate that the resonance frequency of the resulting ESA $(k a \sim 0.63)$ remains the same as found for the unmodified version. The surface current distributions and their orientation along the $X$-axis on the EAD NFRP elements mimic those displayed in Figs. 1(a) and 1(b); and as its 3D directivity pattern shows in Fig. 1(c), it too radiates simply in an electric dipole mode.



(a)

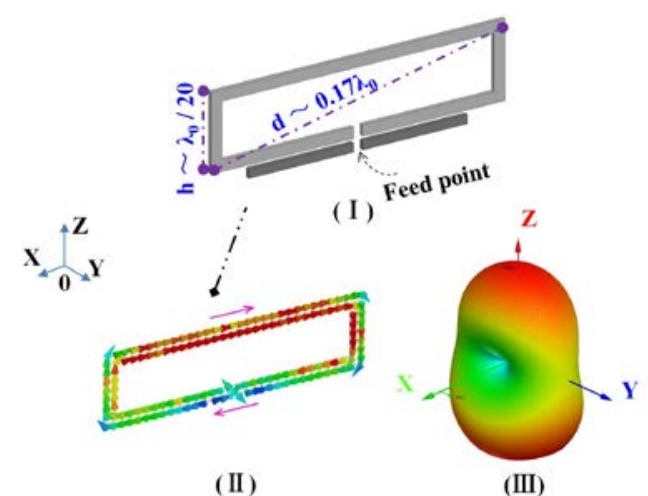

(b)

Fig. 2 The geometries, surface current distributions, and 3D directivity patterns for two magnetic-based dipole antennas: (a) dual CLLs, and (b) single CLL.

\section{B. Electrically small magnetic dipole antenna}

The CLL element is now a common magnetic metamaterial unit cell inclusion that has been widely applied in the microwave field [48, 49]. It has also been used as a NFRP element that is either electrically or magnetically coupled to the driven dipole element to achieve antennas with electrically small size, high radiation efficiency, and good impedance matching to a $50 \Omega$ source without any external matching network [9], [22], [50]. In order to obtain a symmetrical magnetic dipole radiation pattern in those cases, two methods have been generally applied. One is the use of a pair of CLL elements with their gaps in a face-to-face arrangement [36]. This configuration cancels out the impact of the asymmetrical current distributions that form on the $X$-oriented segments of the single CLL element shown in Fig. 2(b), i.e., the currents on the upper strip are stronger than those on the lower strip. One observes in Fig. 2(a) that the two inner strips are close to each other and their currents flow in opposite directions. The same is 
true for the driven dipole arrangement which is taken from [36]. Consequently, the result is a cancellation of their effects. The net behavior then is an effective loop mode, which creates the magnetic dipole behavior. As inferred from Fig. 2(a), this magnetic dipole is oriented along the $Y$-axis. The other approach is the integration of a ground strip or ground plane with the CLL element. Because of the resulting image effect, this configuration is effectively equivalent to that shown in Fig. 2(a) [50].

It is clear when comparing Figs. 2(a) and 2(b) that if the asymmetry of the current distributions along the $X$-axis in the single CLL case is not handled properly, then the expected magnetic dipole response is not obtained. Rather, it will act more like an electric dipole oriented along the $X$-axis and, thus, the desired Huygens source will not be attained. On the other hand, notice that the positioning of the driven dipole strips is changed from Fig. 2(a) to Fig. 2(b). While it does not eliminate the asymmetrical current effects, the latter arrangement will accommodate the electric dipole-driven, single CLL element configuration when the EAD elements are present and will also facilitate recovery of the magnetic dipole radiation performance necessary for the Huygens source. This design is considerably different from the well-known single or double magnetic loop antennas [46]. This single CLL NFRP element is not directly connected with the excitation source nor is it driven by a loop antenna. Rather, it acts as a NFRP element in the presence of the driven dipole element. Furthermore, it enables the resulting antenna system to be nearly completely matched to the source at a much lower frequency, without any external matching circuit [9]. This outcome is quite important because if only one CLL element is used as the NFRP element, the total electrical size $(\mathrm{ka})$ and height is reduced, respectively, from 0.72 to 0.53 and from $\lambda_{0} / 10$ to $\lambda_{0} / 20$. Consequently, the single CLL element version witnesses a reduction of approximately $26.4 \%$ in its electric size and $50 \%$ in its height.

As a remaining design step, it is thus critical to equalize the magnitudes of the top and bottom $X$-axis directed currents in the single CLL NFRP element when it is integrated with the modified EAD ESA to realize the desired Huygens source. This target performance will be realized with a customized arrangement of the CLL and EAD NFRP elements. These features of the design and its associated operational mechanisms will be discussed in Section III (C).

\section{HuYgens SOURCE ANTENNA}

\section{A. Design principles}

According to existing design principles [35]-[37], [44], a broadside radiating Huygens source antenna would be obtained if its electric and magnetic dipoles (NFRP elements) are perpendicularly oriented (e.g., in the $\mathrm{XOY}$-plane), have the same excitation magnitude, and have overlapping phase centers. Then, the main-beam direction of the resulting antenna would be vertical to those dipoles (e.g., along the $Z$-axis). The basic design concept for two orthogonal orientations of the individual dipoles is illustrated in Fig. 3.

It must be emphasized that the goal in any Huygens source ESA design is to achieve electric and magnetic dipoles having the same excitation magnitude. The associated FTBR behavior is illustrated in Table I as a function of the ratio of the excitation magnitudes between the electric and magnetic dipoles. These FTBR values are based on the combined analytic far-field expressions [36], [46] for orthogonally oriented electric and magnetic dipoles. While straightforward in principle, this goal is difficult to achieve in practice with an electrically small package [35].


Fig. 3 Orthogonal design concepts of a Huygens source antenna that radiate along the $+Z$-axis as their broadside direction [35], [40], [41], [44], [46]. The electric and magnetic fields due to electric dipole are labeled: $\mathrm{E}^{\mathrm{e}}, \mathrm{H}^{\mathrm{e}}$; those due to the magnetic dipole are labeled: $E^{\mathrm{m}}, \mathrm{H}^{\mathrm{m}}$. The resulting Huygens source electric and magnetic fields are labeled: $\mathrm{E}^{\mathrm{H}}, \mathrm{H}^{\mathrm{H}}$.

TABLE I. THE FTBR VALUE AS A FUNCTION OF THE RATIO OF THE EXCITATION MAGNITUDES OF THE ELECTRIC AND MAGNETIC DIPOLES

\begin{tabular}{|c|c|c|c|c|c|c|c|}
\hline Ratio & 1 & 1.5 & 2 & 5 & 10 & 50 & $+\infty$ \\
\hline FTBR $(\mathrm{dB})$ & $+\infty$ & 19.91 & 15.31 & 8.36 & 5.69 & 2.47 & 0 \\
\hline
\end{tabular}

\section{B. Antenna design and performance characteristics}

The EAD and CLL NFRP elements can be strategically combined as shown in Fig. 4. Both NFRP elements, as well as the coax-fed dipole are constructed with Rogers Duroid ${ }^{\mathrm{TM}} 5880$ sheets. The sheets containing the EAD element and the dipole antenna are positioned parallel to each other. The sheet containing the CLL element is arranged between the two pieces of the EAD element and orthogonal to and centered on the dipole element. The geometrical centers of the EAD and CLL elements are almost overlapping. The structural parameters for the optimized design are given in Table II.

It is noted that this design is analogous to, but simper than, the one reported in [44]. There, two and four CLL elements with lumped capacitors combine to form the requisite magnetic dipole behavior, while the EAD element is unmodified but includes lumped inductors to achieve the corresponding electric dipole response. In contrast, only one CLL element is used in the present design. As noted previously, this will facilitate a lower Q-factor value and, hence, larger fractional bandwidth for the entire antenna system.

The coax-fed dipole is printed on the bottom surface (towards the source) of a $0.7874 \mathrm{~mm}$ (31 mil) thick 5880 sheet that lies in the $X O Y$ plane. The EAD NFRP element is printed on the top surface of another $0.7874 \mathrm{~mm}$ (31 mil) thick 5880 sheet also lying parallel to the $X O Y$ plane. It produces the field of an electric dipole centered on the $X$-axis. The CLL NFRP element lies in the ZOX plane on a $0.508 \mathrm{~mm}$ (20 mil) thick 5880 sheet. As will be described in Section III(C), it is designed to produce 
the field of a magnetic dipole oriented along the $Y$-axis in the presence of the modified EAD NFRP element. The rectangular gap shown in Fig. 4(c) between the two pieces of the EAD NFRP element is $33.4 \mathrm{~mm} \times 0.508 \mathrm{~mm}$. It is centered along the length of the dipole antenna. The Duroid board on which the magnetic element is printed is centered in this gap and, hence, on the dipole antenna.

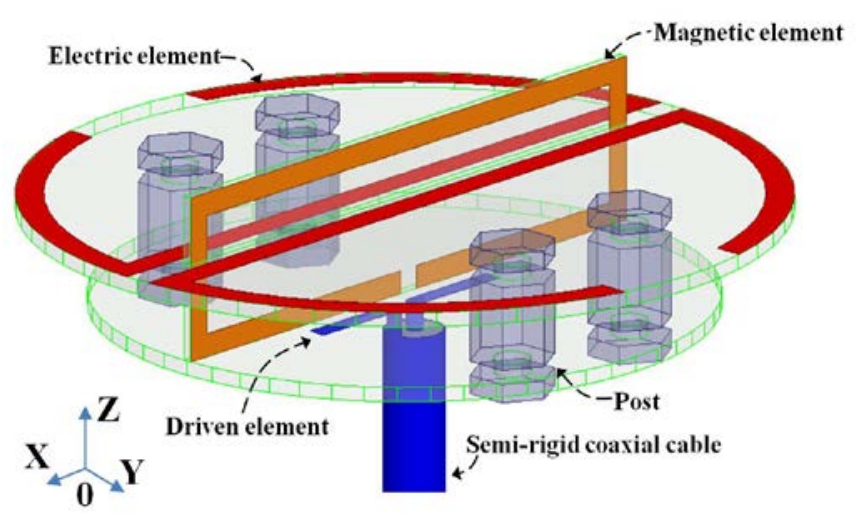

(a)

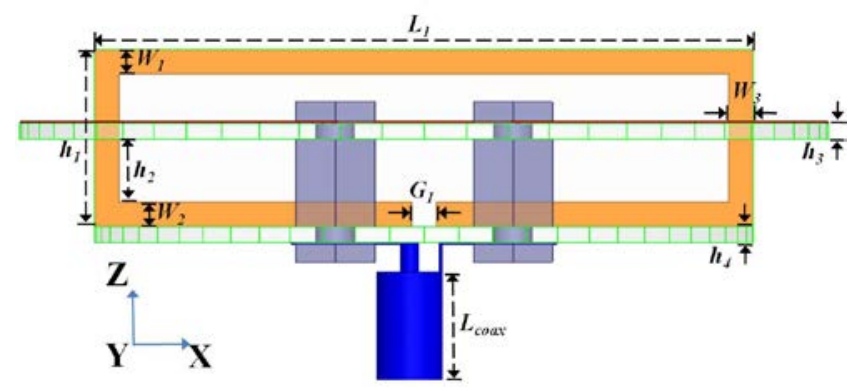

(b)

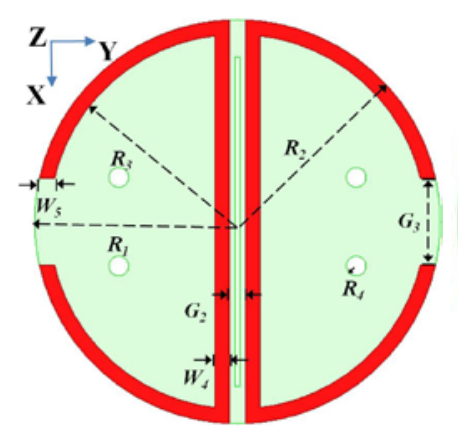

(c)

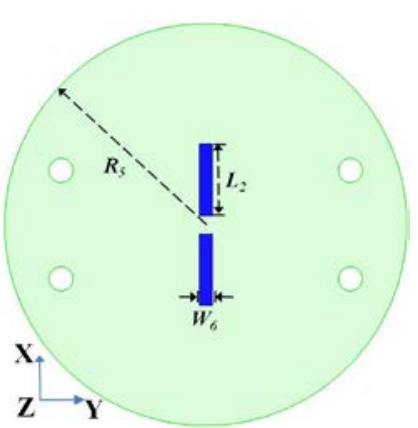

(d)
Fig. 4 The basic configuration of the electrically small, low-profile Huygens source. (a) 3D isometric view, (b) side view, (c) top view of the electric element, and (d) bottom view of the driven dipole element.

TABle II. The Optimized Design Parameters of the Huygens SourCe ANTENNA (ALl DimENSIONS ARE In MiLLIMETERS)

\begin{tabular}{|c|c|c|c|c|}
\hline$L_{1}=33.4$ & $L_{2}=6$ & $L_{\text {coax }}=3$ & $W_{1}=1.25$ & $W_{2}=1.25$ \\
\hline$W_{3}=1.25$ & $W_{4}=1.5$ & $W_{5}=1.55$ & $W_{6}=1$ & $R_{1}=20.5$ \\
\hline$R_{2}=20.5$ & $R_{3}=19$ & $R_{4}=1$ & $R_{5}=16.7$ & $h_{1}=9$ \\
\hline$h_{2}=4.545$ & $h_{3}=0.787$ & $h_{4}=0.787$ & $G_{1}=1.25$ & $G 2=1.6$ \\
\hline$G_{3}=8.8$ & \multicolumn{4}{|c|}{ Null } \\
\hline
\end{tabular}

We note that both the EAD and CLL configurations were not modified to achieve the Huygens source design. Moreover, the short coaxial cable illustrated in Fig. 4 was included with a 3 $\mathrm{mm}$ length in our simulation models to increase their accuracy. Furthermore, to ensure the mechanical stability of the antenna system during the installation and experimental processes, the two parallel substrates are connected with two pairs of hexagonal plastic nuts and screws. They are modeled in the simulations as vertical posts that pass through the two substrates. The vertical board is connected and fixed in place to the top of the dipole antenna board with $\mathrm{AB}$ cure adhesive. As found in our simulation studies, the presence of the posts degrades the radiation efficiency and the ease of impedance matching of the antenna system. Consequently, in the optimized final design, the posts were taken to be the smallest ones that could be purchased. They had R4 $=1.0 \mathrm{~mm}$.

A prototype of this optimized design was fabricated, installed, and measured in one of the University of Electronic Science and Technology of China (UESTC) anechoic chambers. The antenna was fed by a KTG 141-50 type, semi-rigid coaxial cable from Shenzhen Kansai Industrial LTD which had a $50 \Omega$ characteristic impedance. The inner and outer conductors of the coaxial cable were vertically connected and soldered to the separated inner ends of the two metallic center strips of the driven dipole element. Photos of it alone and in the measurement chamber as the antenna under test (AUT) are shown in Fig. 5.

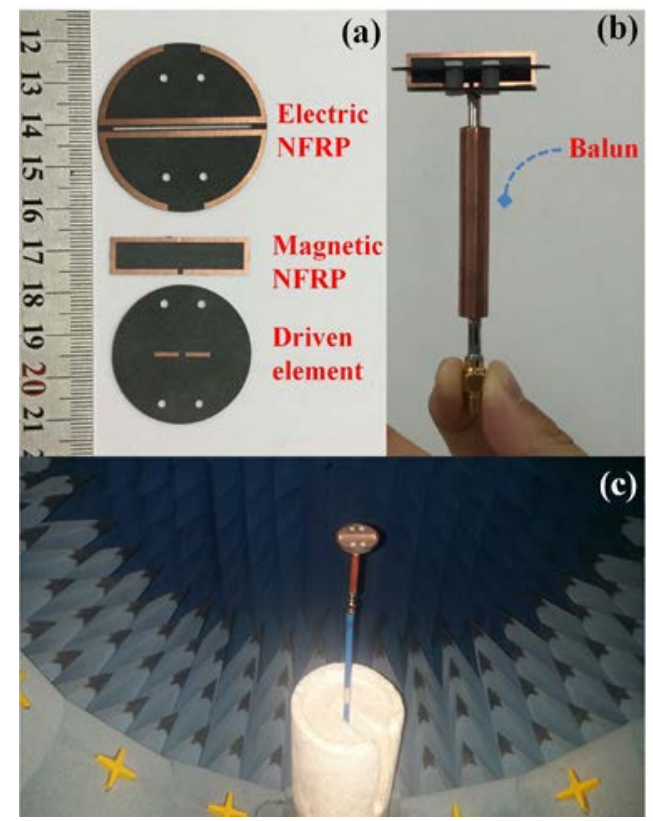

Fig. 5 The fabricated prototype of the electrically small, low-profile Huygens source antenna is shown with the sleeve balun. (a) front and back views before installation, (b) side view after installation, and (c) 3D isometric view of the AUT in the anechoic measurement chamber.

It is worth mentioning that the shorter cable included in the simulations is simply to model the actual transition from coax-line structure to the unbalanced dipole. According to those results, when the length of the feedline $\left(\mathrm{L}_{\text {coax }}\right)$ is longer than $60 \mathrm{~mm}$, relatively strong surface currents appear on its 
metallic outer wall. This effect (1) decreases the peak realized gain and FTBR values $\sim 0.1 \mathrm{~dB}$ and $\sim 0.67 \mathrm{~dB}$, respectively, and (2) makes the radiation pattern tilt away from the broadside direction $3.0^{\circ}$ in the $E$ (ZOX) plane while having little impact on the radiation pattern in the $H(\mathrm{Z} 0 \mathrm{Y})$ plane. In order to eliminate these undesirable effects, a one-quarter wavelength sleeve balun was designed to choke those measurement induced surface currents. It was attached on the feedline close to the antenna as shown in Fig. 5(b) [24], [51], [52].

The $\left|S_{11}\right|$ values as a function of the excitation frequency of the antenna were measured first using an Agilent E8361A PNA vector network analyzer (VNA). The measured results together with the corresponding simulation values are shown in Fig. 6. In order to emphasize the contribution of the balun on the antenna performance, the simulated $\left|S_{11}\right|$ values when the antenna is attached to a shorter coax feedline ( $3 \mathrm{~mm}$ ), and when it is attached to a longer coax feedline $(60 \mathrm{~mm})$ are also plotted in Fig. 6. Comparing these results, it is readily observed that, when the coaxial cable is $3 \mathrm{~mm}$, the antenna is well matched to the $50 \Omega$ source at $f_{0}=1.502 \mathrm{GHz}$ with $\left|\mathrm{S}_{11}\right|_{\min }=-19.41 \mathrm{~dB}$. It has a $-10 \mathrm{~dB}$ impedance bandwidth equal to $8.5 \mathrm{MHz}$.



Fig. 6 Simulated and measured $\left|S_{11}\right|$ values versus the excitation frequency for the forward radiating Huygens source antenna, together with the simulated 3D directivity pattern at the resonance frequency, $1.502 \mathrm{GHz}$, for the $3.0 \mathrm{~mm}$ coaxial feedline case.

The differences in the $\left|\mathrm{S}_{11}\right|$ values in the neighborhood of $f_{0}$ for the longer coax cable arise from a certain amount of leakage current that appears on its outer wall, which in turn leads to field energy being radiated into undesirable directions. While $f_{0}$ essentially remains the same, this change in the load causes the $\left|\mathrm{S}_{11}\right|_{\min }$ value to increase. Moreover, the reflection coefficient does not go to one at frequencies above $f_{0}$. When the antenna and its feedline were integrated with the sleeve balun, those leakage currents were significantly reduced. As a consequence, the measured impedance matching performance was quite similar to that obtained for the shorter-balun simulation case. In particular, the simulation (measurement) results were $\left|\mathrm{S}_{11}\right|_{\min }=$ $-17.69 \mathrm{~dB}(-16.38 \mathrm{~dB})$ at $f_{0}=1.502 \mathrm{GHz}(1.496 \mathrm{GHz})$ and the corresponding -10dB impedance bandwidth was $8.1 \mathrm{MHz}(9.3$ $\mathrm{MHz}$ ). Thus, the total height and electrical size at $f_{0}$ of the measured (simulated) Huygens source antenna system were, respectively, $\lambda_{0} / 20.37=0.0491 \lambda_{0}\left(\lambda_{0} / 20.45=0.0489 \lambda_{0}\right)$ and $k a=0.645(0.642)$. These values confirm that the prototype Huygens source antenna was low-profile and electrically small. Moreover, the measured $-10-\mathrm{dB}$ fractional bandwidth is $0.62 \%$, 10.36 times larger than that reported in [44].

Furthermore, our simulation studies show that if only the electric NFRP element is present, the resonance frequency of the antenna is $f_{0}=1.519 \mathrm{GHz}$ and its peak directivity equals $2.37 \mathrm{dBi}$. On the other hand, if it only has the magnetic NFRP element, its resonance frequency is $f_{0}=1.605 \mathrm{GHz}$ and its peak directivity equals $2.21 \mathrm{dBi}$. Since the Huygens source resonance frequency is $f_{0}=1.502 \mathrm{GHz}$ and its peak directivity is $4.70 \mathrm{dBi}$, this organic combination of the two NFRP elements red shifts the resonance frequency (because of the presence of the additional capacitance that occurs between them) and it significantly increases (nearly doubles) the peak directivity.

The far-field radiation performance characteristics of the Huygens source antenna were studied analytically, numerically and experimentally. The experiments were carried out in an anechoic chamber at UESTC, which incorporates an Agilent N5230A PNA-L VNA and a SATIMO passive measurement system. The simulated and measured normalized radiation patterns obtained at their resonance frequencies are compared with their analytical versions in Fig. 7. It is clear in all three cases that the antenna exhibits good broadside radiation performance. Their main-beam directions are all oriented along the $+Z$-axis, except for the measured case which has a slight tilt away from it: $\sim 3.5^{\circ}$, in the Z0X $(E-)$ plane and $\sim-0.5^{\circ}$ in the $\mathrm{ZOY}(\mathrm{H}-)$ plane. Because of the consistency of the numerical results, we believe this tilt was introduced simply by the measurement set-up.

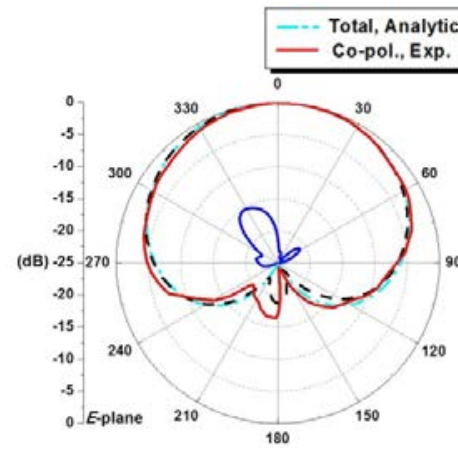

(a)

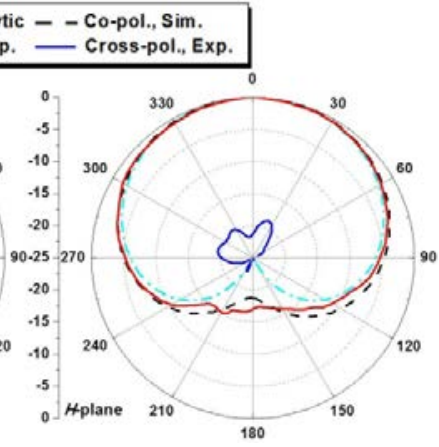

(b)
Fig. 7 Normalized radiation patterns in the ZOX (E-) and ZOY $(H-)$ planes of the Huygens source antenna at resonance frequency centers $(1.502 \mathrm{GHz}$ in simulation and $1.496 \mathrm{GHz}$ in experiment).

Specifically, the simulated (measured) peak realized gain value was $3.30 \mathrm{dBi}(2.03 \mathrm{dBi})$ and the FTBR value was 18.76 $\mathrm{dB}(16.92 \mathrm{~dB})$ at $f_{0}=1.502 \mathrm{GHz}(1.496 \mathrm{GHz})$. The realized gain values correspond to a radiation efficiency of only $R E=$ $71.60 \%$. The reasons for this modest value will be explained below. The half-beam coverage was from $-67^{\circ}$ to $63^{\circ}\left(-62^{\circ}\right.$ to $\left.68^{\circ}\right)$ in the $E$-plane and from $-71^{\circ}$ to $75^{\circ}\left(-70.5^{\circ}\right.$ to $\left.70^{\circ}\right)$ in the $H$-plane. These were both quite close to the idealized analytical values: from $-65.5^{\circ}$ to $65.5^{\circ}$. Moreover, the simulated maximum directivity (strictly along $+Z$-axis) was $4.70 \mathrm{dBi}$, which was very close to the maximum analytical value: 4.77 
$\mathrm{dBi}$ [36]. The quite low experimental cross-polarization levels in the E-plane (lower than $-15.22 \mathrm{~dB}$ ) and in the H-plane (lower than $-18.81 \mathrm{~dB}$ ) indicate that the prototype antenna has a relatively high polarization purity. Note that because the maximum cross-polarization levels in both planes in the simulations were lower than $-32.7 \mathrm{~dB}$, they are not plotted here.

After analyzing the experimental results, the main reasons for the differences in the simulated and measured behaviors have been traced to small dimension errors incurred during the fabrication and installation processes. Because the modified Egyptian axe and single CLL NFRP elements in the antenna design, as well as the sleeve balun, are all narrow-bandwidth resonant devices, these small variations have an impact on the results. For instance, the measured larger impedance bandwidth and the corresponding lower peak realized gain values are further indicators that there were additional losses, which can be ascribed to those errors.

\section{Operational mechanisms}

In order to more clearly explain the operational mechanisms of the broadside radiating, low-profile Huygens source antenna, the surface current distributions on both NFRP and the driven elements at its resonance frequency at different excitation phases (times) are shown in Fig. 8. The directions of these surface currents are highlighted with the additional solid (referring to the magnetic element) and dotted (referring to the electric element) arrows. One clearly sees that the presence of both NFRP elements does not prevent either of them from being excited by the driven dipole element and that these currents mimic their distributions when they are considered separately. With the observed currents on the EAD NFRP element, it will indeed radiate as an electric dipole along the $X$-axis. On the other hand, the single CLL NFRP element continues to have the asymmetry in its currents on its upper and lower horizontal strips. What has happened with the combination that makes it radiate as the requisite magnetic dipole oriented along the $Y$-axis?

First, notice in the integrated design that the CLL NFRP element is now closer to the driven dipole than is the EAD one. Thus, it is more strongly coupled to the driven dipole. This explains the presence of the very strong current distribution on its upper horizontal segment for all the illustrated phase values. Second, because the CLL NFRP element is placed between the pieces of the EAD NFRP element, it is also strongly capacitively coupled to them. Thus, the phase of the CLL currents can be modified by those pieces. Third, as is also apparent from Fig. 8, the EAD NFRP element is now closer to the upper horizontal segment of the CLL NFRP element than to its lower one. Because of this additional capacitive coupling, the currents on the upper surface of the center segments of the EAD pieces and those on the upper segment of the CLL element become out-of-phase with each other. Consequently, the net effect of the current on the upper segment of the CLL element is reduced by the current on the EAD pieces and is thus brought into balance with the lower segment. Notice that the relative phases of the currents on the upper and lower surfaces of the EAD pieces are most strongly influenced, respectively, by the upper horizontal segment of the CLL element and the upper surface of the driven dipole element. Nonetheless, the couplings between all these elements control the phase of the currents and, hence, the strengths of the net responses. For instance, the currents on the lower surface of the center segments of the EAD pieces are oriented out-of-phase to that of the currents on the upper surface for the times: T/4 and 3T/4. In summary, because of these two influential factors: the near-field presence of the EAD pieces and all of the consequent strong couplings between all of the elements, the undesirable pattern in Fig. 2(b) due to the asymmetrical current on the single CLL response is transformed into the desired magnetic dipole along the $Y$-axis shown in Fig. 2(a).

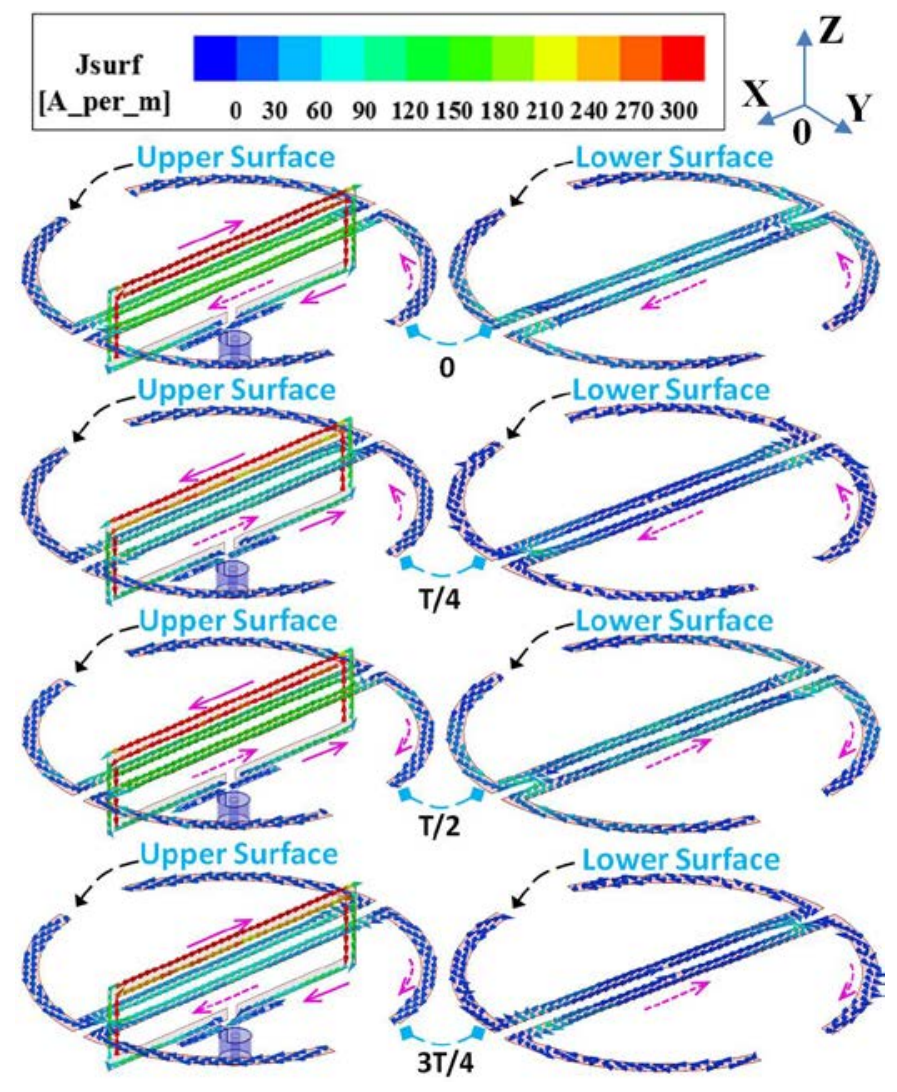

Fig. 8 Surface current distributions for several excitation phases (i.e., times, $\mathrm{T}$ being the period: $T=1 / f_{0}$ ) on the upper surface (left) and lower surface (right) of the EAD and CLL NFRP elements and the driven dipole antenna for the forward radiating Huygens source antenna at its resonance frequency. Note that for better observation of the surface currents, the substrates and posts were made to be transparent.).

Having investigated these physical coupling mechanisms, it was found that one sensible design procedure would be as follows. Step 1: construct a CLL-based NFRP ESA (similar to that in Fig. 2(b)) and determine its operational frequency. Step 2: add a modified EAD NFRP element perpendicular to it and ensure that its phase center overlaps that of the CLL element. Step 3: shift the EAD resonance frequency to be close to that of the CLL element. Step 4: adjust the mutual coupling levels between the EAD and CLL NFRP elements to equalize, as closely as possible, the magnitudes of their radiated powers.

To understand what aspect of the tested prototype design has reduced the radiation efficiency to only $\mathrm{RE}=71.60 \%$ from many of the previously NFRP ESAs which exceed $90 \%$ [9], we re-simulated the design removing the various losses. First, 
simulating the antenna without the balun gives $\mathrm{RE}=73.19 \%$. The $1.59 \%$ reduction due to the balun is a small effect. Second, the impact of the electric properties of the various NFRP elements was studied. These details of those results are presented in Table III. On one hand, by making the dielectrics lossless, the RE values improved only a little ( 1\%). Similarly, by only making the EAD pieces lossless, we again witnessed little change: a $1.74 \%$ improvement. On the other hand, there was a significant improvement $(16.67 \%)$ when the magnetic element was made lossless. Clearly, the copper loss associated with the magnetic element plays the most important role in decreasing the antenna's radiation efficiency. As shown in Fig. 8 , this makes sense because the largest surface currents are present on it.

TABLE III. IMPACT OF THE MATERIAL PROPERTIES ON THE RADIATION EFFICIENCY OF THE HUYGENS SOURCE ANTENNA

\begin{tabular}{|c|c|c|}
\hline PFRP element & $\begin{array}{c}\tan \delta=0 \\
\text { (substrate) }\end{array}$ & PEC \\
\hline Only changing electric element & $74.08 \%$ & $74.93 \%$ \\
\hline Only changing magnetic element & $74.81 \%$ & $89.86 \%$ \\
\hline Changing both elements & $75.70 \%$ & $92.85 \%$ \\
\hline No change & \multicolumn{2}{|c|}{$73.19 \%$} \\
\hline
\end{tabular}

\section{BACKWARD RADiATING HUYGENS SOURCE ANTENNA}

In order to further clarify the radiation mechanisms of the broadside radiating low-profile, Huygens source ESA design, another version that radiates in the opposite direction (along the -Z-axis) was obtained. The design follows the understanding gained from Fig. 9. Simply, by changing the phase on one of the dipole elements (i.e., either the electric or magnetic dipole by choice) by about $\pi=\mathrm{T} / 2$, the Huygens source can be made to radiate in the $-Z$-direction.

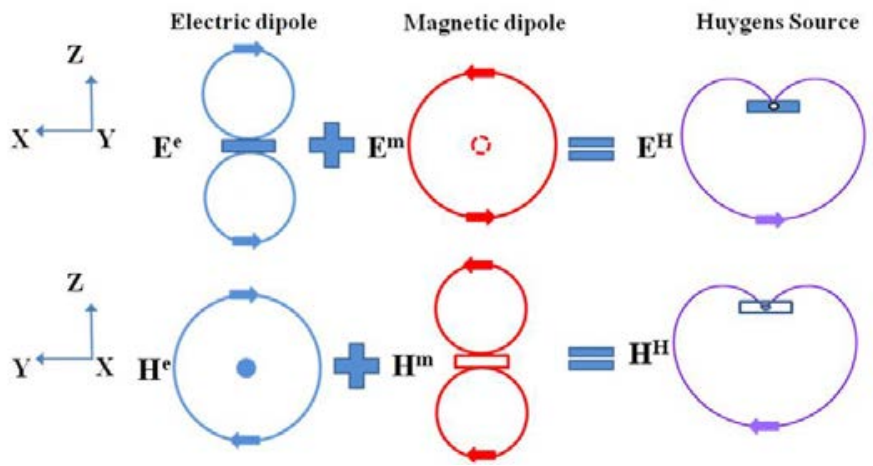

Fig. 9 Design concept of the Huygens source antenna with broadside radiation along the -Z-axis [35], [40], [41], [44], [46].

Consequently, the same configuration shown in Fig. 4 was modified slightly to achieve the desired backward radiating response. The optimized design parameters are listed in Table IV. As expected from the results reported in [44] and as will be demonstrated below, the relative locations in height of the two NFRP elements provided the necessary phase change of the currents.
The simulated results for the optimized design are presented in Figs. 10-12. As is shown in Fig. 10, the antenna is well matched to the $50 \Omega$ source at the resonance frequency $f_{0}=$ $1.505 \mathrm{GHz}$ with $\left|\mathrm{S}_{11}\right|_{\min }=-16.63 \mathrm{~dB}$; its $-10 \mathrm{~dB}$ impedance bandwidth is equal to 7.6 MHz. Its electrical size at $f_{0}$ is $k a=$ 0.670. It exhibits excellent backward radiation at $f_{0}$ with a maximum directivity of $4.71 \mathrm{dBi}$ along the $-Z$-axis, and a 17.09 dB FTBR. Its peak realized gain and radiation efficiency are, respectively, $3.10 \mathrm{dBi}$ and $70.44 \%$.

TABLE IV. OPTIMIZED DESIGN PARAMETERS OF THE BACKWARD RADIATING Huygens SOURCE ANTENNA (All Dimensions ARE IN MiLlimeters)

\begin{tabular}{|c|c|c|c|c|}
\hline$L_{1}=35.6$ & $L_{2}=6$ & $L_{\text {coax }}=3$ & $W_{1}=1.25$ & $W_{2}=1.25$ \\
\hline$W_{3}=1.25$ & $W_{4}=1.5$ & $W_{5}=1.55$ & $W_{6}=1$ & $R_{1}=21.25$ \\
\hline$R_{2}=21.25$ & $R_{3}=19.75$ & $R_{4}=1$ & $R_{5}=17.8$ & $h_{1}=8.1$ \\
\hline$h_{2}=3.75$ & $h_{3}=0.787$ & $h_{4}=0.787$ & $G_{1}=1.45$ & $G_{2}=1.8$ \\
\hline$G_{3}=12$ & \multicolumn{4}{|c|}{ Null } \\
\hline
\end{tabular}

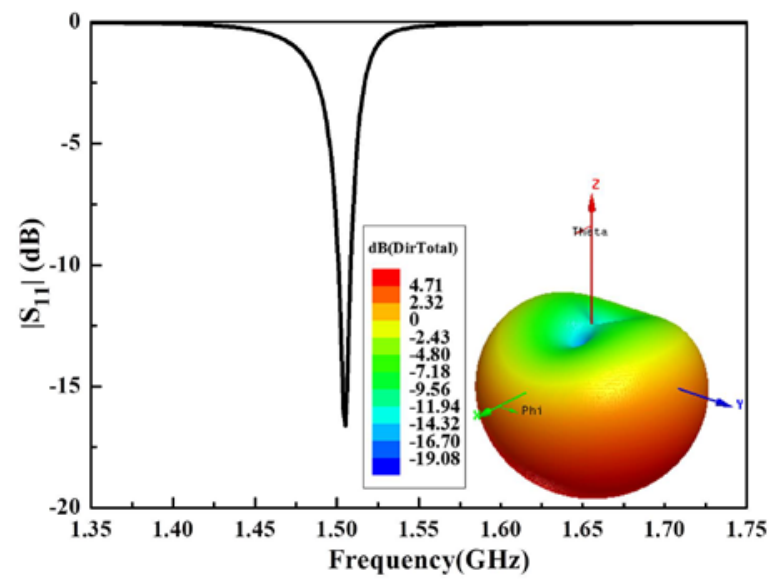

Fig.10 Simulated $\left|\mathrm{S}_{11}\right|$ values versus the excitation frequency for the backwards radiating Huygens source antenna, together with its 3D directivity pattern at its resonant frequency $f_{0}=1.505 \mathrm{GHz}$.



(a)

(b)

Fig. 11 Normalized radiation patterns in the E- and H-planes for the backwards radiating Huygens source antenna at its resonance frequency $f_{0}=1.505 \mathrm{GHz}$.

Also, the simulated 2-D normalized radiation patterns, together with the analytical ones, are presented in Fig. 11. The simulated half-beam widths in $E$ - and $H$-planes are, respectively, in the interval from $115^{\circ}$ to $245^{\circ}$ and from $108^{\circ}$ to $254^{\circ}$, which is close to the analytical range: $114.5^{\circ}$ to $245.5^{\circ}$. 
Because the maximum cross-polarization levels in both of these planes are lower than $-44.3 \mathrm{~dB}$, they were not plotted.

The surface current behaviors on the metallic elements of the antenna are shown in Fig. 12. In general, they appear to be the same as those with the forward radiating cases shown in Fig. 8. They are for the CLL NFRP element. Hence, the phase of the "magnetic dipole" does not change. However, the currents on the lower surface of the EAD NFRP are not. They have an opposite phase behavior. This means the phase of the "electric dipole" currents witnesses the requisite $\pi$ shift. Therefore, the combined "magnetic dipole" and "electric dipole" elements construct the Huygens source which radiates along the $-Z$-axis as expected from Fig. 9.

Additionally, notice that the current orientations on the four arc-shape parts of the upper surface of the EAD pieces are not in step with those on the two straight segments. This is clearly seen in the $\mathrm{T} / 4$ and $3 \mathrm{~T} / 4$ cases for the forward radiation version in Fig. 8, and similarly for the 0 and T/2 cases for the backward radiation version in Fig. 12. Nevertheless, they always are oriented in step with those on the corresponding lower surfaces. This phenomenon indicates that the upper surface currents on the four arc-shape parts of the EAD pieces are mainly induced by the driven element, instead of the CLL element. Again, this behavior is opposite to the forward directed design.

Finally, as was already pointed out in Table III, the copper loss from the CLL element has the most significant impact on the antenna efficiency. As seen in Fig. 12, a very strong current is again concentrated on its upper horizontal strip. Also note that in all of the cases discussed so far, the widths of each strip of the CLL element were the same, i.e., $W_{1}=W_{2}=W_{3}$. Having realized the detrimental behavior of the upper strip after the prototype testing, we decided to explore numerically a possible way to improve it using this backward radiating case. Following the concept in [53], we studied what the impact would be of only increasing the width $W_{1}$ on the upper horizontal strip to decrease the current density on it and, hence, to balance it even more with the current density on the lower one. The strip width was increased from $1.25 \mathrm{~mm}$ to $2.0 \mathrm{~mm}$ and the antenna was retuned. We found that its radiation efficiency improved up to $80.92 \%$ (a 10.48\% improvement) with only moderate changes to the other performance characteristics. In detail, the $\left|\mathrm{S}_{11}\right|_{\min }=-20.65 \mathrm{~dB}$ at $f_{0}=1.63$ $\mathrm{GHz}$ with a $-10 \mathrm{~dB}$ impedance bandwidth equal to $10.7 \mathrm{MHz}$. The electrical size $k a=0.70$ and its maximum directivity was $4.68 \mathrm{dBi}$ strictly along the $-Z$-axis with a FTBR $13.41 \mathrm{~dB}$. With this initial success, other modifications of the CLL element are currently under consideration to achieve further improvements of the radiation efficiency.

\section{CONCLUSIONS}

A simple, electrically small, low-profile, Huygens source ESA that radiates primarily into its broadside direction has been presented in this paper. It consists of two NFRP elements, i.e., a modified (split) EAD element and a single CLL element. Antennas that were constructed with each of these NFRP elements were first introduced to understand their individual radiation mechanisms and performance characteristics. In the combined design, the EAD (CLL) NFRP element produced the electric (magnetic) dipole required for the Huygens source behavior. The capacitive coupling between these NFRP elements was utilized to eliminate the noted disadvantage of the single CLL element antenna, which had seriously degraded its magnetic dipole response. The design of the forward radiating Huygens source ESA was obtained and explained. A prototype version was fabricated and tested. The measurement results were in good agreement with the corresponding analytical and simulated values. A backward radiation version was also designed. It was used to further explain the radiation mechanisms of both designs. It was also used successfully to explore initial considerations to improve the radiation efficiency of both designs. Given the very good broadside radiation performance characteristics in spite of its low profile and electrically small size, the reported simple Huygens source ESA can be easily scaled to work at different frequencies for many practical applications, including precise transponders, sensor devices, GPS, RFID and wireless power transfer systems.

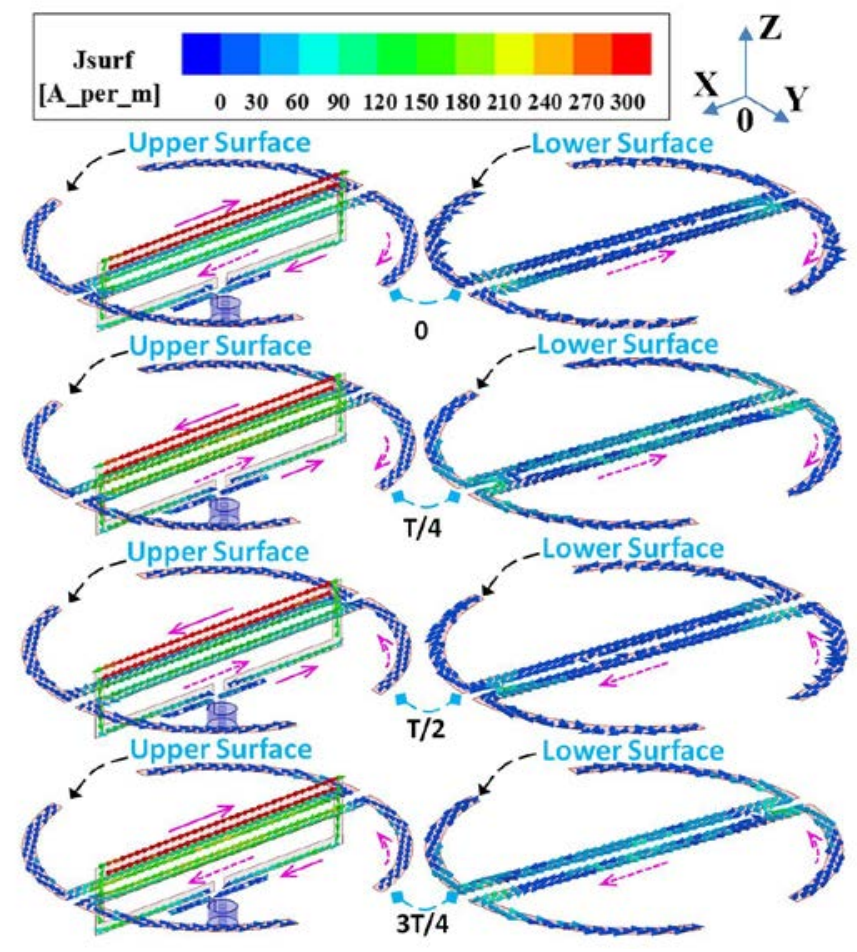

Fig. 12 Surface current distributions on the upper surface (left) and lower surface (right) of Egyptian axe, and on the CLL and driven elements of the antenna with backward radiation performance characteristics at $f_{0}$, with different excitation phases.

\section{References}

[1] S. Lim, R. L. Rogers, and H. Ling, "A tunable electrically small antenna for ground wave transmission,” IEEE Trans. Antennas Propag., vol. 54, no. 2, pp. 417-421, Feb. 2006.

[2] J. L. Volakis, and K. Sertel, "Narrowband and wideband metamaterial antennas based on degenerate band edge and magnetic photonic crystals," Proc. IEEE, vol. 99, no. 10, pp. 1732-1745, Oct. 2011.

[3] Y. Dong and T. Itoh, "Metamaterial-based antennas," Proc. IEEE, vol. 100, no. 7, pp. 2271-2285, Jul. 2012.

[4] J. Oh, and K. Sarabandi, "Low profile vertically polarized Omnidirectional wideband antenna with capacitively coupled parasitic element," IEEE Trans. Antennas Propag., vol.62, no. 2, pp. 977- 982, Feb. 2014.

[5] M. M. H. Armanious, S. V. B. Olaya, J. S. Tyo, M. C. Skipper, M. D. 
Abdalla, L. L. Altgilbers, and A. Bryan, "An electrically small conical folded dipole antenna for use as a compact, self-resonant mesoband high-power microwave source," IEEE Trans. Antennas Propag., vol.62, no. 12, pp. 5960- 5967, Dec. 2014.

[6] J. Zhang, and Y. Long, "A novel metal-mountable electrically small antenna for RFID tag applications with practical guidelines for the antenna design,” IEEE Trans. Antennas Propag., vol.62, no. 11, pp. 5820- 5829, Nov. 2014.

[7] S. R. Best, "Electrically small resonant planar antennas: Optimizing the quality factor and bandwidth,” IEEE Antennas Propag. Mag., vol. 57, Iss. 3, pp. 38-47, Jun. 2015.

[8] M. Hosseini, D. M. Klymyshyn, G. Wells, and X. Liu, "Short and open circuited EBG resonator antennas: miniaturization with a shorting plate and dielectric loading," IEEE Trans. Antennas Propag., vol. 63, no. 10, pp. 4305-4314, Oct. 2015.

[9] R. W. Ziolkowski, P. Jin, and C.-C. Lin, "Metamaterial-inspired engineering of antennas,” Proc. IEEE, vol. 99, no. 10, pp. 1720-1731, Oct. 2011.

[10] R. F. Harrington, "On the gain and beamwidth of directional antennas," IEEE Trans. Antennas Propag., vol. 6, no. 3, pp. 219-225, Jul. 1958.

[11] R. F. Harrington, "Effect of antenna size on gain, bandwidth and efficiency,” J. Res. Nat. Bur. Stand, vol. 64-D, pp. 1-12, Jan./ Feb. 1960.

[12] Y. Yamada, W. Hong, W. Jung, and N. Michishita, "High gain design of a very small normal mode helical antenna for RFID tags," in Proc. IEEE TENCON 2007, Taipei, Taiwan, Oct.30-Nov.2, 2007, pp. 1-4.

[13] W. Hong, Y. Yamada, and N. Michishita, "Low profile small normal mode helical antenna achieving long communication distance," in Proc. 2008 International Workshop on Antenna Technology (iWAT2008), Chiba, Japan, March 4-6, 2008, pp.167-170.

[14] P. Hajizadeh, H. R. Hassani, and S. H. Sedighy, "Planar artificial transmission lines loading for miniturization of RFID printed quasi-Yag antenna,” IEEE Antenna Wireless Propag. Lett., vol. 12, pp. 464-467, 2013

[15] A. S. A. Jalal, A. Ismail, A. R. H. Alhawari, M. F. A. Rasid, N. K. Noordin, and M. A. Mahdi, "Miniaturized metal mount Minkowski fractal RFID tag antenna with complementary split ring resonator," Progress In Electromagnetics Research C, vol. 39, 25-36, Apr. 2013.

[16] T. Ungan, M. Freunek, M. Muller, W. D. Walker, and L. M. Reindl, "Wireless energy transmission using electrically small antennas," in Proc. 2009 IEEE Radio and Wireless Symposium, San Diego, CA, , Jan. 2009, pp. 526-529.

[17] I.-J. Yoon and H. Ling, "Realizing efficient wireless power transfer using small folded cylindrical helix dipoles," IEEE Antennas Wireless Propag. Lett., vol. 9, pp. 846-849, 2010.

[18] D. S. Ricketts, M. J. Chabalko, and A. Hillenius, "Experimental demonstration of the equivalence of inductive and strongly coupled magnetic resonance wireless power transfer," Appl. Phys. Lett., vol. 102, no. 5, 053904, Feb. 2013.

[19] Z. Popovic, E. A. Falkenstein, D. Costinett, and R. Zane, "Low-power far-field wireless powering for wireless sensors,” Proc. IEEE, vol. 101, no. 6, 1397-1409, Jun. 2013.

[20] H. Mosallaei and K. Sarabandi, "Antenna miniaturization and bandwidth enhancement using a reactive impedance substrate," IEEE Trans. Antennas Propag., vol. 52, no.9, pp. 2403-2414, Sep. 2004.

[21] F. Yang and Y. Rahmat-Samii, Electromagnetic Band Gap Structures in Antenna Engineering, Cambridge, UK: Cambridge University Press, 2009.

[22] P. Jin, and R. W. Ziolkowski, "High-directivity, electrically small, low-profile near-field resonant parasitic antennas,” IEEE Antennas Wirel. Propag. Lett., vol. 11, pp. 305-309, 2012.

[23] M.-C. Tang, R. W. Ziolkowski, "Efficient, high directivity, large front-to-back-ratio, electrically small, near-field-resonant-parasitic antenna,” IEEE Access, vol. 1, no. 1, pp. 16 - 28, May 2013.

[24] M.-C. Tang, and R. W. Ziolkowski, "A study of low-profile, broadside radiation, efficient, electrically small antennas based on complementary split ring resonators," IEEE Trans. Antennas Propag., vol. 61, no. 9, pp. 4419 - 4430, Sep. 2013.

[25] E. E. Altshuler, T. H. O’Donnell, A. D. Yaghjian, and S. R. Best, "A monopole superdirective array,” IEEE Trans. Antennas Propag., vol. 53, no.8, pp. 2653-2661, Aug. 2005.

[26] A. D. Yaghjian, T. H. O’Donnell, E. E. Altshuler, and S. R. Best, "Electrically small supergain end-fire arrays," Radio Sci., vol.43, no. RS3002, pp. 1-13, 2008.

[27] S. Lim, and H. Ling, "Design of a closely spaced, folded Yagi antenna,"
IEEE Antennas Wirel. Propag. Lett., vol. 5, pp. 302-305, 2006

[28] T. Kokkinos and A. P. Feresidis, "Electrically small superdirective endfire arrays of metamaterial-inspired low-profile monopoles," IEEE Antennas Wirel. Propag. Lett., vol. 11, pp. 568-571, 2012.

[29] A. Noguchi, and H. Arai, "3-element super-directive endfire array with decoupling network," in Proc. 2014 International Symposium on Antennas and Propagation (ISAP), Kaohsiung, Taiwan, Dec. 2-5, 2014.

[30] Z. Bayraktar, P. L. Werner, and D. H. Werner, "The design of miniature three-element stochastic Yagi-Uda arrays using particle swarm optimization,” IEEE Antennas Wirel. Propag. Lett., vol. 5, pp. 22-26, 2006.

[31] M.-C. Tang, R. W. Ziolkowski, S. Xiao, and M. Li, “A high-directivity, wideband, efficient, electrically small antenna system,” IEEE Trans. Antennas Propag., vol. 62, no. 12, pp. 6541 - 6547, Dec. 2014.

[32] O. S. Kim, S. Pivnenko, and O. Breinbjerg, "Superdirective magnetic dipole array as a first-order probe for spherical near-field antenna measurements," IEEE Trans. Antennas Propag., vol. 60, no. 10, pp.4670 - 4676, Oct. 2012.

[33] A. D. Yaghjian, "Increasing the supergain of electrically small antennas using metamaterials," in Proc. 2009 European Conferenc on Antennas and Propagation (EuCAP), Berline, Germany, Mar. 2009, pp. 858-860.

[34] A. Haskou, A. Sharaiha, and S. Collardey, "Design of small parasitic loaded superdirective end-fire antenna arrays," IEEE Trans. Antennas Propag., vol. 63, no. 12, pp. 5456 - 5464, Dec. 2015.

[35] S. R. Best, "Progress in the design and realization of an electrically small Huygens source," in Proc. 2010 International Workshop on Antenna Technology (iWAT), Lisbon, Portugal, Mar. 1-3, 2010

[36] P. Jin and R. W. Ziolkowski, "Metamaterial-inspired, electrically small Huygens sources," IEEE Antennas Wirel. Propag. Lett., vol. 9, pp. 501-505, 2010.

[37] T. Niemi, P. Alitalo, A. O. Karilainen, and S.A. Tretyakov, "Electrically small Huygens source antenna for linear polarization," IET Microw. Antennas Propag., vol. 6, Iss. 7, pp. 735-739, 2012.

[38] P. Alitalo, A. O. Karilainen, T. Niemi, C. R. Simovski, and S. A. Tretyakov, "Design and realisation of an electrically small Huygens source for circular polarization," IET Microwaves, Antennas \& Propagation, vol. 5, no. 7, pp. 783-789, 2011.

[39] M. Pigeon, C. Delaveaud, L. Rudant, and K. Belmkaddem, "Miniature directive antennas," Int. J. Microw. Wirel. T., vol. 6, no. 1, pp. 45-50, Feb. 2014

[40] K.-M. Luk and B. Wu, "The magnetoelectric dipole: A wideband antenna for base stations in mobile communications," Proc. IEEE, vol. 100, no. 7, pp. 2297-2307, Jul. 2012.

[41] H. J. Seo, and A. A. Kishk, "Wideband linear and dual-polarized antenna based on Huygens source principle," in 2011 XXXth URSI General Assembly and Scientific Symposium, Istanbul, 2011, pp. 1-4.

[42] M. Li and K.-M. Luk, "A low-profile, low-backlobe and wideband complementary antenna for wireless application,” IEEE Trans. Antennas Propag., vol. 63, no. 1, pp. 7-14, Jan. 2015.

[43] H.-J. Seo, and A. A. Kishk, "Wideband magnetic-electric antenna with linear single or dual polarization," Progress In Electromagnetics Research (PIER), vol. 155, pp. 53-61, Mar. 2016.

[44] R. W. Ziolkowski, "Low profile, broadside radiating, electrically small Huygens source antennas," IEEE Access, vol. 3, pp. 2644-2651, Dec. 2015.

[45] ANSYS/ANSOFT High Frequency Structure Simulation (HFSS), ver. 13.0, ANSYS Corp. [Online]. Available at www.ansoft.com.

[46] C. A. Balanis, Antenna Theory: Analysis and Design, 3rd edition, New York: Wiley Interscience, 2005.

[47] M.-C. Tang and R. W. Ziolkowski, "Two-element Egyptian axe dipole arrays emphasizing their wideband and end-fire radiation performance," IET Microw. Antennas Propag., vol. 9, no. 13, pp. 1363 - 1370, Oct. 2015.

[48] N. Engheta and R.W. Ziolkowski, Metamaterials: Physics and Engineering Explorations, Piscataway, N.J.: IEEE-Wiley Press, 2006.

[49] T. J. Cui, D. R. Smith, and R. Liu, Metamaterials: Theory, Design, and Applications, New York, Springer, 2009.

[50] P. Jin and R. W. Ziolkowski, "Multi-frequency, linear and circular polarized, metamaterial-inspired, near-field resonant parasitic antennas," IEEE Trans. Antennas Propag., vol. 59, no. 5, pp. 1446 - 1459, May 2011.

[51] S.A. Saario, J.W. Lu and D.V. Thiel, "Full-wave analysis of choking characteristics of sleeve balun on coaxial cables," Electron. Lett., vol.38, no.7, pp.304-305, Mar. 2002.

[52] C. Icheln, J. Krogerus, and P. Vainikainen, "Use of balun chokes in 
small-antenna radiation measurements," IEEE Trans. Antennas Propag., vol.53, no. 2, pp.498-506, Apr. 2004.

[53] A. K. Horestani, C. Fumeaux, S. F. Al-Sawari, and D. Abbott, "Split ring resonators with tapered strip width for wider bandwidth and enhanced resonance," IEEE Microw. Comp. Lett., vol. 22, no. 9, pp. 450-452, Sept. 2012

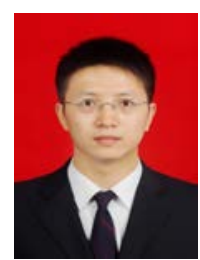

Ming-Chun Tang (S'12-M'13-SM'16) received the B. S. degree in physics from the Neijiang Normal University, Neijiang, China, in 2005 and the Ph. D. degree in radio physics from the University of Electronic Science and Technology of China (UESTC), in 2013. From August 2011 to August 2012, he was also with the Department of Electrical and Computer Engineering, The University of Arizona, Tucson, AZ, USA, as a Visiting Scholar. He is currently an Assistant Professor in the College of Communication Engineering, Chongqing University, China. His research interests include electrically small antennas, RF circuits, metamaterial designs and their applications.

Prof. Tang was a recipient of the Best Student Paper Award in the 2010 International Symposium on Signals, Systems and Electronics (ISSSE2010) held in Nanjing, China. He is serving as a reviewer for IEEE/IET journals including the IEEE Transactions on Microwave Theory and Techniques, IEEE Transactions on Antennas and Propagation, IEEE Antennas and Wireless Propagation Letters, IEEE Antennas and Propagation Magazine, IET Microwaves, Antennas \& Propagation, and Electronics Letters.

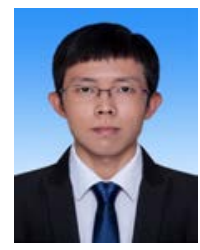

Hao Wang (S'16) received the B.S. degree in communication engineering from the Lanzhou University (LZU), Lanzhou, China, in 2014.He is currently pursuing the M.S. degree in electronic science and technology at Chongqing University, Chongqing, China.

His research interests include electrically small antennas, planar antennas and arrays.

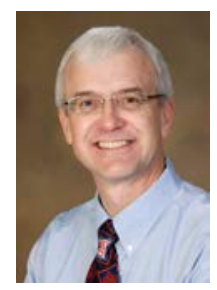

Richard W. Ziolkowski (M'87-SM'91-F'94) received the B.Sc. (magna cum laude) degree (Hons.) in physics from Brown University, Providence, RI, USA, in 1974; the M.S. and Ph.D. degrees in physics from the University of Illinois at Urbana-Champaign, Urbana, IL, USA, in 1975 and 1980, respectively; and the Honorary Doctorate degree from the Technical University of Denmark, Kongens Lyngby, Denmark in 2012.

He was the Australian DSTO Fulbright Distinguished Chair in Advanced Science and Technology from 2014 to 2015. He was the Computational Electronics and Electromagnetics Thrust Area Leader with the Lawrence Livermore National Laboratory, Engineering Research Division, before joining The University of Arizona, Tucson, AZ, USA, in 1990. He is currently the Litton Industries John M. Leonis Distinguished Professor with the Department of Electrical and Computer Engineering and a Professor with the College of Optical Sciences, The University of Arizona. He is also a Distinguished Professor with the Global Big Data Technologies Centre, University of Technology Sydney, Ultimo NSW, Australia. His current research interests include the application of new mathematical and numerical methods to linear and nonlinear problems dealing with the interaction of electromagnetic and acoustic waves with complex linear and nonlinear media, as well as metamaterials, metamaterial-inspired structures, and applications-specific configurations.

Prof. Ziolkowski is a Fellow of the Optical Society of America (OSA). He served as the President of the IEEE Antennas and Propagation Society in 2005. He is also actively involved with the URSI, OSA and SPIE professional societies. 